\title{
Effect of Donepezil Hydrochloride on Cognitive Function Recovery of Rats With Alzheimer's Disease
}

\author{
Qiliang Zhou ${ }^{1}$, Siping Nie ${ }^{2 *}$, Zhihuo Liu ${ }^{1}$ \\ ${ }^{1}$ Department of Human Anatomy and Embryology, Changsha Medical \\ University, Changsha, Hunan 410219, P.R. China; \\ ${ }^{2}$ School of Public Health, Guizhou Medical University, Guiyang, Guizhou \\ 550004, P.R. China.
}

First Author: Qiliang Zhou, Associate professor, Changsha Medical University, P.R. China. Email: 1223796992@qq.com.

${ }^{*}$ Corresponding Author: Siping Nie, professor, Guizhou Medical University, P.R. China. Email: niesiping-nsp@163.com.

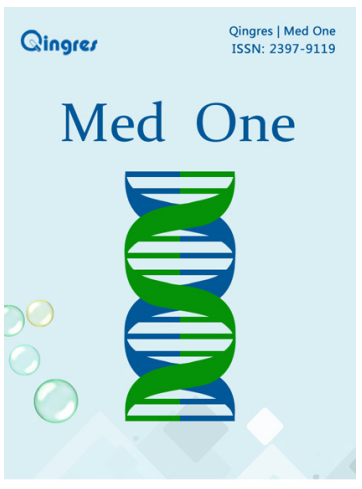

http://mo.qingres.com

\section{GOPEN ACCESS}

DOI: 10.20900/mo.20160023

Received: July 18, 2016

Accepted: September 20, 2016

Published: October 25, 2016

Copyright: $\odot 2016$ Cain et al. This is an open access article distributed under the terms of the Creative Commons Attribution License, which permits unrestricted use, distribution, and reproduction in any medium, provided the original author and source are credited.

\begin{abstract}
Objective: To explore the effect of donepezil hydrochloride on cognitive function recovery of rat with Alzheimer's disease and the positive expression of nitric oxide synthase and acetylcholinesterase (NOS and AchE) in the brain tissues of rats with Alzheimer's disease (AD).
\end{abstract}

Methods: Forty SD (Sprague Dawley) rats were randomly classified into a model group, a treatment group, a control group, and a sham operation group. Each group had 10 rats. AD rat models were prepared with $A \beta 1-40$. Donepezil hydrochloride was orally administered to rats in the treatment group two weeks after modeling. Rat recognition function was assessed. The positive expression of NOS and AchE in rat brain tissues was observed via immunohistochemistry after administration of 28 days.

Results: Rat escape latency was significantly longer after modeling $(p<0.010)$. Compared to the model group, treatment group escape latency was significantly shortened and the difference was significant $(p<0.05)$. NOS and AchE positive expression mainly reflects in hippocampus region tissue. Compared with the control, and sham, operation groups, NOS positive expression decreased and AchE positive expression increased in the model group. The difference was statistically significant $(p<0.01)$. Compared with the model group, NOS expression in the treatment group increased and AchE expression decreased. The difference was significant $(p<0.01)$.

Conclusion: Donepezil hydrochloride can promote $A D$ rat cognitive 
function recovery. Its effects are also associated with increased NOS protein expression in hippocampus neuron cells in addition to specifically inhibiting the AchE activities.

Key Words: Donepezil hydrochloride; Rats; Alzheimer's disease

\section{INTRODUCTION}

Alzeimer's disease (AD) is a degenerative neurosis characterized mainly by cognitive and memorial dysfunctions. It is a common dementia among the elderly. AD is now the fourth most common elderly disease that causes death after cancer, heart and cerebrovascular diseases. It seriously reduces patient quality of life and is a heavy burden families and society. The association of learning and memory impairment of $A D$ patients with the brain cholinergic dysfunction is known. Donepezil hydrochloride is a selective inhibitor of cholinesterase.

A large number of clinical studies proved that Donepezil can significantly improve AD patient cognitive and memory functions and it was considered an effective drug for treat AD patients [1-2]. Therefore, it is of an important significance to positively explore the possible functional mechanism of donepezil hydrochloride except to inhibit the cholinesterase.

In this study, $A D$ model rats were the research subject to study the effect of donepezil hydrochloride on NOS protein expression in hippocampal neurons and its effect on positive AchE expression in AD model rat brain tissue. The results are reported as follows.

\section{MATERIALS}

\subsection{Experimental Animals}

40 SD male rats, weight $200 \pm 20 \mathrm{~g}$ from the Hunan SJA Laboratory Animal Co., Ltd., holding license numbered: SCXK (Hunan) 2009-0004.

\subsection{Experimental Drug}

Donepezil hydrochloride tablets (Shaanxi Ark Pharmaceutical Co., Ltd., National medicine permission number: H20030583). Daily dosage (5 $\mathrm{mg}$ ) for $60 \mathrm{~kg}$ adult, the equivalent dosage for rats was $0.5 \mathrm{mg} / \mathrm{kg}$. It was prepared as a suspension of $0.05 \mathrm{mg} / \mathrm{mL}$ with the distilled water.

\subsection{Reagents and Instrument}

Amyloid $\beta$-protein 1-40 (A $\beta 1-40)$, NOS, and AchE polyclonal antibodies purchased from Beijing Bioss Biotechnology Co., Ltd; PV9000 kit and DAB chromogenic agent were purchased from Beijing ZSGB Biotechnology Co., Ltd.; Morris water maze and brain stereotaxic apparatus (Shenzhen RWD Life Science Co., Ltd); Finesse 325 paraffin slicing machine (British Shando Corporation); B x 51 optical microscope and IPP6.0 image analysis system (Japanese Olympus Corporation).

\section{METHODS}

\subsection{Animal Grouping and Administration}

All rats were fed for one week. They were then divided into a control group, sham operation group, model group, and a treatment group (model + Donepezil hydrochloride). There were 10 rats in each group. The treatment group was administered orally with a donepezil hydrochloride suspension of $0.5 \mathrm{mg} / \mathrm{kg}$ two weeks after the modeling. The sham operation, and model, groups were administered normal saline. The control group was fed normally. They were administered once a day, for 28 continuous days.

\subsection{Model Preparation (Modeling)}

$A D$ rat models were prepared with $A \beta 1-40$ in References ${ }^{[3]}$. It was anesthetized with $10 \%$ chloral hydrate $(3 \mathrm{~mL} / \mathrm{kg})$ and fixed on the stereotaxic apparatus. A scalpel cut an incision of $1.5 \mathrm{~cm}$ long along the anterior median line of the disinfected area. The bregma was located and adjusted and orient it with the stereotaxic apparatus to ensure the syringe needle would be aligned $2 \mathrm{~mm}$ on the right side of the bregma and $3.5 \mathrm{~m}$ behind the bregma. This is the drill point. Drill and open the skull, slowly push the syringe needle at a speed of $0.3 \mathrm{~mm} / \mathrm{min}$ to $2.7 \mathrm{~mm}$ into the skull at precisely the hippocampus. A $1-40$ was diluted into $5 \mathrm{ug} / \mathrm{uL}$ with sterile normal saline and cultivated at $37^{\circ} \mathrm{C}$ to aggregate it. $A \beta 1$ 40 of $5 \mathrm{ug} / \mathrm{uL}$ was slowly injected to the rat with a microinjector of $1 \mathrm{uL}$ at the injection speed of approximately $0.1 \mathrm{uL} / \mathrm{min}$. Upon completing the injection, the needle was removed at a speed of 0.3 $\mathrm{mm} / \mathrm{min}$ and the skull sealed with dental mud and 
skin suturing. After completing the operation, rat was subject to a conventional feeding and intramuscular injection of penicillin against infection. 1uL of normal saline was injected into the hippocampus of rat in the sham operation group and the operation method was identical to that in model group.

\subsection{Evaluation of Rat Learning and Memory}

A Morris water maze was used to test learning and memory abilities. For one week after modeling and three weeks after drug administration, rats were subject to a water maze exercise of for 7 days, once in the morning and the afternoon. On day 7, a place of entry was selected to put the rats into the water facing the pond wall to observe and record the time required for rats to find and climb onto the platform, namely, escape latency.

\subsection{NOS and AchE Immunohistochemistry testing}

After completing administration, all were anesthetized with chloral hydrate and brain matter was harvested. Tissue was fixed in $4 \%$ paraformaldehyde for 24 hours. Brain tissues were dehydrated with gradient alcohol, hyalinized with xylene, infiltrated and embedded by paraffin, and sliced into 5 um thick coronal sections. Sections were incubated in $3 \%$ hydrogen peroxide for $10 \mathrm{~min}$, and washed for three times with 0.0 M PBS. They were then heated to boiling for $30 \mathrm{~s}$. Tissue was washed three times with $0.01 \mathrm{M}$ PBS. Brain tissue from all groups was dropped with $50 \mathrm{uL}$ of diluted rabbit anti-rat NOS polyclonal antibody (1:100) and rabbit anti-rat AchE polyclonal antibody (1:100), respectively. The primary antibody in rats in negative control was replaced by $0.01 \mathrm{M}$ PBS. They were incubated $2 \mathrm{~h}$ at $37^{\circ} \mathrm{C}$ and washed three times with $0.01 \mathrm{M}$ PBS. They were dropped with the goat anti-rabbit of PV 9000 kit biotin labeled goat anti-rabbit, incubated for 30min at $37^{\circ} \mathrm{C}$ and washed with three times with $0.01 \mathrm{M}$ PBS. They were dropped with PV 9000 kit horseradish peroxidase labeled streptavidin, incubated for 20min at $37^{\circ} \mathrm{C}$ and washed three times with $0.01 \mathrm{M}$ PBS. They were dropped with DAB developer to develop the color for $10 \mathrm{~min}$ at a room temperature and washed with distilled water to terminate the reaction. The sections were then stained with hematoxylin stain for $10 \mathrm{~min}$ and washed in running water for $5 \mathrm{~min}$ They were then subjected to color separation for 10 s in $0.5 \%$ hydrochloric acid alcohol separation solution and washed in running water for $2 \mathrm{~min}$. The sections were hyalinized with xylene and gradient alcohol and sealed with neutral resin. Three sections (slices) were taken from each brain tissue for each indicator. Each section was placed under the microscope 400 times to randomly select five views without repeated positive expressions. Positive cells of the same unit area were counted using the IPP 6.0 image processing system.

\subsection{Statistical Analysis}

SPSS19.0 statistical software was used for data analysis. All data are expressed by $x \pm s$, and the single factor analysis of variance was used for comparison among groups. LSD method was used for homogeneity of variance, and Tambane's T2 method was used for heterogeneity of variance. $p<$ 0.05 indicates the statistically significant difference.

\section{RESULTS}

\subsection{Learning and memory by group}

The tests of escape latency via the Morris water maze show a significant extension of escape latency in the model, and treatment, groups before administration. The difference is statistically significant compared with the results of the control, and sham operation, groups $(p<0.01)$. Compared to the model group, treatment group latency is significantly shortened after administration $(p<0.05)$. See Table 1.

Table 1. Group Escape Latency before, and after, administration: a comparison $(x \pm s, s)$

\begin{tabular}{llll}
\hline Group & $\mathbf{n}$ & Before Administration & After Administration \\
\hline Control & 10 & $38.26 \pm 6.33$ & $37.18 \pm 5.92$ \\
Sham Operation & 10 & $37.57 \pm 7.02$ & $36.50 \pm 6.09$ \\
Model & 10 & $92.65 \pm 16.431^{\prime}$ & $65.29 \pm 13.711^{\prime}$ \\
Treatment & 10 & $90.72 \pm 17.801^{\prime}$ & $44.04 \pm 10.222^{\prime}$ \\
\hline
\end{tabular}

Note: 1 . Compared with control group and sham operation group, $p<0.01 ; 2$. Compared with model group, $p<0.05$. 


\subsection{NOS and AchE Positive Expression by Groups}

NOS and AchE positive expression by group was detected using immunohistochemistry. A chocolate brown, or brownish yellow, stain in neuronal cell cytoplasm, or protrusions, are positive. The main expression area is in hippocampus. NOS expression in the model group decreased while
AchE expression increased, indicating a statistically significant difference compared with the control, and sham operation, groups $(p<0.01)$. After drug administration, NOS expression in the treatment group increased and AchE expression decreased, which indicates a statistically significant difference compared with the model group $(p<0.01)$. (Table 2 and Fig. 1 and 2).

Table 2. NOS and AchE Positive Expression by Group ( $\pm \pm s$, piece/mm2)

\begin{tabular}{llll}
\hline Group & $\mathbf{n}$ & NOS & AchE \\
\hline Control & 10 & $116.85 \pm 21.431^{\prime}$ & $96.36 \pm 19.271^{\prime}$ \\
Sham Operation & 10 & $107.83 \pm 19.611^{\prime}$ & $103.52 \pm 24.851^{\prime}$ \\
Model & 10 & $59.52 \pm 13.09$ & $177.50 \pm 35.43$ \\
Treatment & 10 & $95.73 \pm 17.301^{\prime}$ & $112.02 \pm 22.351^{\prime}$ \\
\hline
\end{tabular}

Notes: Compared with model group, $p<0.01$;

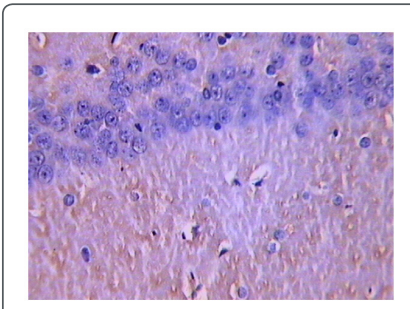

Control Group

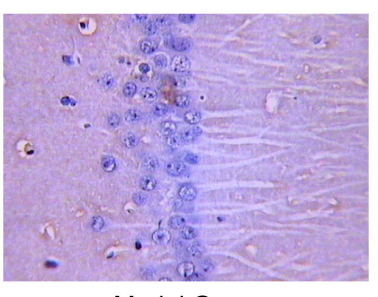

Model Group

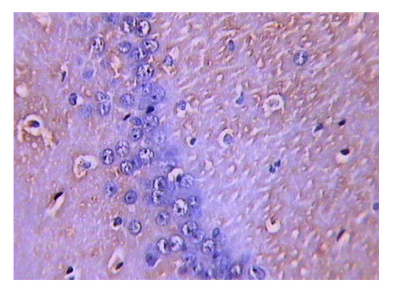

Sham Operation Group

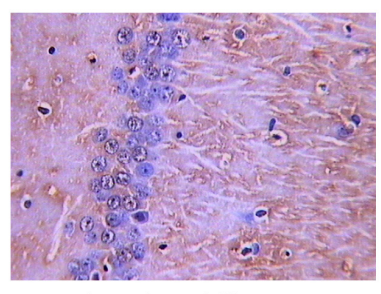

Treatment Group

Fig. 1 NOS Positive Expression in Rat Brains of Various Groups (×400)

\section{DISCUSSION}

Currently $A D$ pathogenesis is unclear. There are many theories concerning its etiology, including: genetics theory, $\beta$-amyloid protein $(A \beta)$ deposition theory, cholinergic theory, inflammatory theory, excitatory amino acid theory, free radical damage theory, and aluminum poisoning theory. AD pathology is characterized by cerebral cortical atrophy,

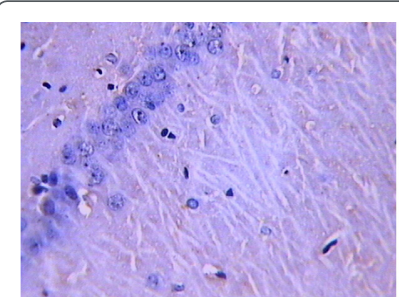

Control Group

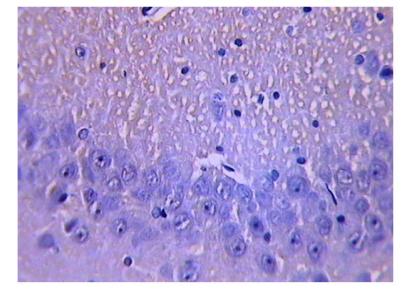

Model Group

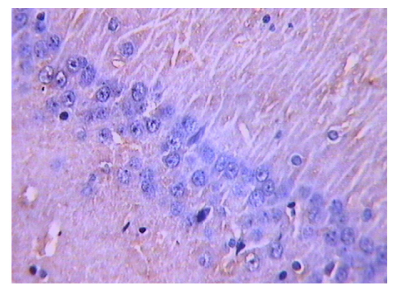

Sham Operation Group

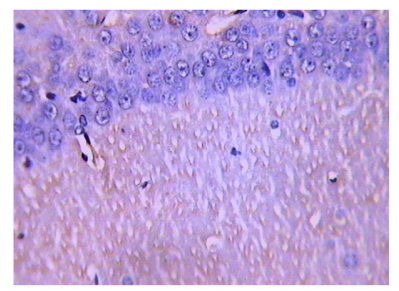

Treatment Group
Fig. 2 AchE Positive Expression by Group (×400)

accompanied by $A \beta$ deposition, neurofibrillary tangles, large decreases in the number of memory neurons, and senile plagues formation. At present, $A \beta$ is generally accepted as the main factor inducing $A D$. The key for $A D$ formation and development of the neuropathological process is that $A \beta$ continuously injures brain cells to lead to a progressive mental 
decline and causing senile plague formation. The $A \beta$ deposition can decrease the choline acetyltransterase activities and cause the reduction of cholinergic neurons around $A \beta$ deposition. This leads to memory impairment. It has been reported that some medications can result in a lessening of Leydig cells and regressive and / or degenerative changes in the epididymis, seminal vesicle, ventral prostate, and coagulating gland in male rats ${ }^{[4]}$. This experiment used the currently accepted method of acute injection of $A \beta$ in the rat brain. It can cause the functional loss of cholinergic neurons and learning and memory abilities dysfunction, and result in $A \beta$ deposition in the brain. Therefore, it is a better animal model. In this experiment, model group escape latency persisted for two weeks after modeling $(p<0.01)$, indicating the decline of learning and memory ability of rats after modeling and a successful modeling.

The current accepted theory attributes $A D$ cognitive symptoms to a shortage of cholinergic neurotransmitters. Acetylcholine (Ach) is an important neurotransmitter in the cholinergic pathway of the central nervous system. It is has widely involved in the cognitive process of in-vivo learning and memory. Learning and memory ability decrease with as Ach levels decrease. Donepezil hydrochloride is a hexaoxypyridine oxide and the generation-II specific reversible central AchE inhibitor. It can increase Ach concentration by inhibiting Ach hydrolysis to improve the cognitive function of $A D$ patients ${ }^{[5]}$. However, Ach is not stable in the body and it hydrolyzes into choline and acetic acid. AchE activity levels can indirectly reflect the Ach level. Some animal studies reported that donepezil hydrochloride had treatment effects on $A \beta$-induced $A D$ rats ${ }^{[6]}$. It could significantly improve $A D$ model rat learning and memory impairment and reduce the injury of free radicals in hippocampus neurons ${ }^{[7]}$. The results of this study showed that donepezil hydrochloride could improve AD rat learning and memory function $(p<0.05)$, indicating that it could inhibit AchE activities in rat brains and AchE positive expression of hippocampal neurons in $A D$ rats was also significantly decreased $(p<0.05)$.

It is currently thought that long-term potentiation (LTP) is the main mechanism of synaptic plasticity, learning and memory. Nitric oxide (NO) can maintain the LTP effect of hippocampal synapses, and it can promote $\mathrm{AcH}$ release and other neurotransmitter releases in the form of super-nitrite in the central nervous system to affect learning and memory ${ }^{[8]}$. NO is considered a key transmitter in learning and memory. NO is not stable in the body. NOS is the key enzyme, rate-limiting enzyme in NO synthesis. Change in this activity can reflect NO level in the body. Studies show that the learning and memory processes of experimental rats may be associated with nNOS activity increases ${ }^{[9]}$. The results of this study showed the increased AchE positive expression and the decreased NOS positive expression in rat hippocampal neuron cells in the model group $(p<0.01)$, indicates a decrease in learning and memory ability of model rats. After the treatment with donepezil hydrochloride, NOS expression in AD rat hippocampal neuron cells increased and the difference was significant compared with model group rats $(p<0.01)$. This suggests that donepezil hydrochloride can improve rat learning and memory abilities by promoting NOS expression and enhancing the level of NO. These studies show that donepezil hydrochloride can improve $A D$ rat learning and memory impairment, and it can also associate with the enhancement of NOS activity in the brain hippocampus and the adjustment of the metabolism of messenger molecular NO in the central nervous system in addition to the inhibition of the AchE activities.

\section{CONFLICT OF INTEREST STATEMENT}

No conflict of interests and financial disclosure is present. Qiliang Zhou contributes the manuscript, Siping Nie provides the technical guide and Zhihuo Liu provides the language editing service.

\section{REFERENCES}

1. Huang L, Fu Y, Li Y. Efficacy observation on old dementia treated with donepezil hydrochloride. Chin Commun Doc. 2011; 13(10): 124-125.

2. Yu B. Clinical efficacy observation on old dementia treated with donepezil. Chin J Mod Drug Appl. 2012; 6(18): 103-104.

3. Zhang X, Liu Z, Liu J, Guo H, Qu H, Peng W, BaoY, Yin L, Song Y. Effect of electroacupuncture on memory hypofunction of Alzheimer's disease rats. Chin J Tradit Chin Med Pharm. 2012; 27(3): 706-709.

4. Agrawal RC, Prachi P. Chemopeventive effects of andographis panniculata extract in vivo and in vitro models. Med One. 2016; 1(4): 2.

5. Li J, Sun R, Ma D, Zhang Y. Clinical study of donepezil hydrochloride in treatment of mildmoderate senile dementia. Chin J Geriatric 
Care. 2006; 4(2): 21-22.

6. Liu H, Xie X, Zou H, Ye L, Liu K. Therapeutic effect of donepezil hydrochloride on $\beta$-amyloidinduced senile dementia in rats. Chin J Geront. 2007; 27(1): 135-137.

7. Li Q, Qi J. Effect of donepezil hydrochloride on learning and memory ability and CAT and GSHPx content in AD model rats. J Qiqihar Med Coll. 2007; 28(1): 25-27.
8. Zhang $Y$, Wu Z, Tian X, Li Y, Li M, Yang $M$. Effects of purging fire and resuscitating prescription on metabolism of nerve messenger molecule NO in spontaneous aged dementia mice. Chin J Trad Med Sci Technol. 2009; 16(4): 300-301.

9. Liu H, Chen J, Tian S, Tan S, Gao Q. The changes and possible role of neuronal nitric oxide synthase in learning and memory. Chin $\mathrm{J}$ Neuroimmunol Neurol. 2000; 7(2): 116-122. 